\title{
Au-delà des genres : décalages stylistiques entre l'anglais et le français
}

\author{
Jacqueline Bossé-Andrieu
}

Université d'Ottawa

\begin{abstract}
Stylistic differences between English and French are minimal since these two languages are relatively close. However, there are indeed some differences that should be taken into account when one has to write in both languages. Contrastive analyses done in translation studies have shown that certain features are specific to each language. This paper will present a number of these shifts between English and French, including word order, the degree of homogeneity between subject and verb, and the modality used to provide information. We will illustrate several of these differences that are not texttype-nor field-specific through various examples drawn from actual nonspecialized explanatory texts.
\end{abstract}

\section{Introduction}

Au Canada, un certain nombre de rédacteurs et de rédactrices sont appelés à rédiger à la fois en anglais et en français. Pour ce faire, il est bon qu'ils connaissent les grandes tendances stylistiques propres à chacune des deux langues, tendances communes à divers types de textes. Même si, à l'heure actuelle, beaucoup de travaux sur le discours portent sur l'étude de textes appartenant à un même genre discursif, il ne nous paraît pas inopportun de montrer que l'anglais et le français privilégient certaines tournures dans les textes non spécialisés, quel que soit le domaine ou le genre auquel ils appartiennent. Les études contrastives, menées dans l'optique de la traduction, éclairent la singularité et les caractéristiques de chacune des deux langues, traits qu'on ne connait souvent qu'intuitivement quand on est unilingue et dont on ne prend vraiment conscience qu'en comparant une langue à l'autre. On découvre alors que certains tours, qui sont naturels dans une langue, le sont moins ou ne le sont pas du tout dans l'autre. Les études menées dans une perspective interlinguistique et souvent à partir d'analyses de corpus se sont multipliées depuis le début des années 80 , quelque 
vingt ans après la parution d'une oeuvre pionnière, la Stylistique comparée du français et de l'anglais de J.-P. Vinay et J. Darbelnet, qui, éditée pour la première fois en 1958, a été traduite en anglais en 1995 . Outre les nombreux textes et articles publiés dans des revues de linguistique appliquée ou de traduction portant sur un point particulier (aspects, coordination, ponctuation, verbes, etc.), plusieurs ouvrages brossent un tableau des divergences entre les deux langues. Mentionnons entre autres les suivants : Syntaxe comparée du français et de l'anglais. Problèmes de traduction (J. GuilleminFlescher, 1981), Approche linguistique des problèmes de traduction (H. Chuquet et M. Paillard, 1987), Relations discursives et traduction (M. Ballard, éditeur, 1995), Linguistique contrastive et traduction (sous la direction de J. Guillemin-Flescher, 1991), Lire et traduire. Anglais français (C. Demanuelli et J. Demanuelli, 1991). D'autres ouvrages pédagogiques fournissent des remarques et des exemples intéressants ${ }^{2}$. Nous présenterons ci-après quelques-unes des tendances stylistiques propres à l'anglais et au français signalées dans ces ouvrages, tendances qu’il faut se garder de confondre avec des règles absolues, mais qu'il faut connaître quand on rédige dans les deux langues. Nous dégagerons les préférences respectives de l'anglais et du français en ce qui touche plus particulièrement : i) l'ordre des mots dans la phrase, ii) l'homogénéité de l'énoncé, c'est-à-dire la relation entre le terme de départ de l'énoncé et le verbe, et iii) la modalisation de l'énoncé. Nous illustrerons ces décalages par la comparaison de textes analogues et authentiques rédigés dans les deux langues. Ces textes, placés en annexe, appartiennent à trois domaines différents ; ils ont tous une visée informative et une portée générale. La première paire est constituée de textes de vulgarisation sur les maladies de l'oeil écrits par des spécialistes ; la deuxième, de préfaces de dictionnaires généraux ; la troisième, de passages extraits de manuels de style. Quant aux courts exemples qui émaillent notre texte, ils appartiennent eux aussi à des domaines différents (avis au public, notice technique, textes publicitaires, etc.) et sont de nature informative.

\section{Organisation de l'énoncé}

En ce qui concerne l'ordre des mots dans la phrase, deux grandes différences séparent l'anglais et le français. Alors que l'anglais a une nette préférence pour l'ordre canonique (sujet + verbe + complément) et pour la coordination, le français, quant à lui, n'hésite pas à réorganiser l'énoncé selon un ordre raisonné qu'il juge logique et préfere, à la coordination, soit la juxtaposition, soit la subordination de certains éléments de l'énoncé. C'est ainsi que, à l'issue de la comparaison de plusieurs traductions de Alice in Wonderland, Ballard (1995, p. 288-9) conclut : 
La traduction de Papy, la plus calquée sur l'anglais, présente des séries de procès posés en succession, celle de Parisot pratique plus souvent l'effacement de la coordination ou l'enchâssement, donnant alors l'impression d'un texte plus construit, avec des relations hiérarchiques entre propositions, plus français à notre avis.

Plusieurs études, dont celles de Ballard (1995) et de Quillard et Akhras (1996) ont, en effet, montré la prédilection de l'anglais pour la coordination et, en ce qui concerne le français, le penchant pour deux stratégies contraires : dans certains cas, le français a recours à la juxtaposition (le lien est alors implicite entre deux éléments de l'énoncé), dans d'autres, à la subordination. Alors que, en anglais, les conjonctions de coordination (en particulier « and ») abondent, en français, on remarque un emploi fréquent des signes de ponctuation, en particulier de la virgule. Demanuelli (1995) lie les différences dans l'emploi de la virgule à la différence dans la démarche syntaxique des deux langues, entre l'anglais qui « semble volontiers adhérer » à l'ordre canonique, préfère la phrase simple et l'ordre chronologique, et le français qui "privilégie les agencements plus souples, sinon plus lâches, et la mise en relief par le biais de l'ordre des mots, autrement dit de l'inversion totale ou partielle ou de l'imbrication " (Demanuelli, 1995, p. 130). Les extraits de l'annexe 1 , tirés de deux ouvrages de vulgarisation sur les troubles de la vision, illustrent ces tendances. On y remarque la prédilection de l'anglais pour l'ordre canonique alors que le français n'hésite pas à réorganiser les phrases, à placer des circonstants en position frontale et à interrompre l'énoncé par des enchâssements (incises et imbrications). Si, dans le texte anglais, la plupart des phrases (onze sur dix-huit) sont du type sujet + verbe + complément, dans le texte français, on trouve plusieurs antépositions adverbiales et adjectivales et des enchâssements, dont plusieurs circonstants en milieu de phrase. Il ressort de la comparaison des deux textes que le texte français donne l'impression d'être plus travaillé, puisqu'on y remarque, entre autres ${ }^{3}$, une réorganisation évidente de l'énoncé par rapport à l'ordre canonique.

Cette réorganisation correspond aux préférences du français en ce qui concerne un ordre qu'il juge logique ${ }^{4}$, rationnel, conforme à une façon raisonnée de présenter les idées. Respectant cet ordre, le français, contrairement à l'anglais, commence rarement une phrase par un élément essentiel, mais par l'élément qui rappelle une information connue du locuteur et préfère terminer la phrase par l'élément d'information important ${ }^{5}$, ce que permet l'antéposition des circonstants. Comme le note Bonnard (1981, p.125), en français, il est logique de placer en tête de phrase les compléments de lieu ou de temps qui constituent " une bonne assise pour le thème de la phrase " et les « compléments marquant un phénomène chronologiquement 
ou logiquement antérieur au fait communiqué ». L'anglais, lui, préfere conserver l'ordre grammatical. Un simple exemple : alors que le français dit "Après la pluie (vient) le beau temps », l'anglais dit "Sunshine comes after the rain. »

La cause étant chronologiquement antérieure au résultat, le français préfère l'exprimer en premier, mais non l'anglais. C'est ainsi que Chassigneux (1991, p. 72), professeur à I'ESIT de Paris, dit " avoir toujours été frappé par les différences d'ordre des mots entre l'anglais et le français ", point qu'il considère comme "l'un des "tests" les plus sûrs d'une bonne traduction ", et donne l'exemple d'une phrase traduite en français qu'il juge peu naturelle car elle suit de trop près la structure de la phrase anglaise de départ et donne d'abord l'effet, puis la cause ${ }^{6}$. Cette remarque rejoint celles de Vinay et Darbelnet (1958, p. 202-3) qui avaient noté la tendance du français à placer en tête de phrase ou avant le verbe les propositions qui ne constituent pas l'élément d'information essentiel, et donc, entre autres, celles qui indiquent la cause, et donné l'exemple suivant :

Sûr d'obtenir gain de cause, il attendit sans inquiétude l'ouverture du procès :

He waited unconcernedly for the opening of the case as he felt sure to win.

Selon ces auteurs, si l'ordre voulant que la cause précède l'effet est possible en anglais, il se rencontre moins fréquemment qu'en français.

Donnons deux autres exemples pour illustrer la différence entre les deux langues en ce qui concerne l'agencement syntaxique. À l'Université d'Ottawa, pendant des travaux de réfection de la bibliothèque, un panneau annonçait :

Pour mieux vous servir, nous effectuons des travaux de rénovation/To serve you better, we are renovating.

En revanche, un autre annonçait :

Montez au deuxième étage si vous voulez vous rendre au pavillon Montpetit/

Walk up to the second floor to go to Montpetit Hall.

Les deux énoncés français surprennent le francophone. En effet, dans le premier cas, le francophone attend l'élément connu et donc non essentiel (les travaux) en position initiale, et donc l'expression du but à la fin de la phrase ; le but mentionné, inconnu du lecteur, est ce que les responsables veulent faire connaître et mettre en relief (pour se faire pardonner les désagréments provoqués par les travaux). Par contre, en anglais, il semble naturel de donner en début de phrase l'élément d'information que l'on veut mettre en relief ( « to serve you better "). ${ }^{7}$ Dans le deuxième exemple, le but ( «se rendre quelque part») est l'élément connu du lecteur; le francophone attend donc la proposition de but en tête de phrase, comme dans la description des 
étapes à suivre pour monter un appareil qui donnent d'abord le but de l'opération puis le moyen d'y parvenir. En anglais, il semble que, même si les ouvrages de style conseillent le contraire ${ }^{8}$, on place plus facilement l'élément important et nouveau en début de phrase.

Ces préférences de l'anglais et du français relativement à l'agencement syntaxique des énoncés expliquent donc un certain nombre de décalages observés dans la comparaison de textes écrits dans les deux langues. D’autres décalages dans la construction de l'énoncé découlent de préférences en ce qui concerne la façon de présenter les événements.

\section{Homogénéité de l'énoncé}

La construction d'un énoncé résulte aussi, comme le notent Chuquet et Paillard (1987, p. 139), du choix d'un terme de départ et d'un prédicat. Ce terme de départ peut être un "animé » - en particulier un «animé humain » ou un terme inanimé, et le syntagme verbal peut contenir un verbe « animé » — désignant « un procès qui renvoie à une activité généralement attribuée à un animé humain » (Guillemin-Flescher, 1981, p. 201), ou un verbe inanimé. Or, les études contrastives montrent que l'anglais respecte plus que le français le principe d'homogénéité de l'énoncé, principe énoncé par Guillemin-Flescher et qui consiste à « mettre en relation des termes dont les référents appartiennent à la même catégorie du réel " (p. 181). Le français, lui, emploie souvent un syntagme nominal point de départ de l'énoncé et un syntagme verbal qui appartiennent à des catégories du réel différentes et n'hésite pas à associer un terme de départ inanimé à un verbe animé. C'est la raison pour laquelle, lorsqu'on traduit des textes du français vers l'anglais, il faut souvent procéder à une certaine « homogénéisation » de l'énoncé. Guillemin-Flescher (1981) donne de nombreux exemples de traductions dans lesquelles des énoncés français contenant un terme de départ inanimé et un syntagme verbal animé sont traduits en anglais par des énoncés homogènes contenant soit un terme de départ animé et un verbe animé, soit un terme de départ inanimé et un verbe inanimé. Citons-en un de chaque type : « Un rire moqueur m'accompagna (J. Green, L'autre, p. 22) " est traduit par «I heard her laugh at me as I walked away (S. Dibben, Paris VII)" (p. 381) ; "Ces voitures ont reçu une isolation phonique particulièrement poussée ... (SNCF, p. 24) » est traduit par "These coaches have particularly efficient sound proofing (éd. angl.) " (p. 383). Il est vrai que cette tendance de l'anglais à « l'homogénéisation dans la représentation du réel »(Guillemin-Flescher, 1981,p. 81) est toute relative puisqu'il est possible en anglais aussi de mettre en relation un sujet inanimé et un verbe qui semble indiquer que le sujet fait une action consciente (ex. : the house looks south/la maison donne au sud). Mais, même si l'anglais ne va pas aussi 
loin que le japonais, langue dans laquelle on ne peut concevoir qu'un sujet inanimé accomplisse une action consciente (Uchiyama, 1991) ${ }^{9}$, il importe de savoir qu'il est courant en français d'associer un terme de départ inanimé et un verbe animé, et que ce procédé est moins fréquent en anglais. Ce décalage se remarque dans la comparaison de textes anglais et français : on note souvent, dans les textes anglais, l'emploi fréquent du verbe « to be ", alors que le français évite souvent le verbe « être " et le remplace par un verbe animé et imagé ${ }^{10}$. Donnons un exemple : dans le texte anglais d'une notice accompagnant un tourne-disque Sanyo, on lit :

Silence is an important part of music : the notes are only part of the music a composer writes. The silence between the notes is just as important.

La traduction française donne :

Le silence constitue une partie importante de la musique et les notes ne sont qu'un des éléments d'une création musicale réalisée par un compositeur.

Entre les notes, le silence joue un rôle essentiel. (C'est nous qui soulignons)

Le français n'hésite donc pas à animer des inanimés et à leur prêter une intention. Ce procédé, comme nous l'avons dit, existe en anglais, mais il est moins fréquent. Notons aussi que cette tendance "animiste" du français est parfois renforcée par son emploi de verbes tels que "venir ", "se mettre à ", " aller ", "vouloir ", verbes adjonctifs " particuliers au français " et « qui s'insèrent tout naturellement dans les phrases les plus usuelles " (Vinay et Darbelnet, p. 206). Ces verbes, qui semblent prêter une volonté au sujet s'emploient avec des sujets humains, même lorsque le sujet est "passif " (comme dans "elle s'est vu décerner la médaille d'or/ il s'est vu forcé de partir "), et aussi avec des sujets inanimés (comme dans "le hasard vient souvent faire échouer les meilleurs projets », exemple tiré du dictionnaire Harraps à l'entrée venir et traduit par "chance often wrecks the best plans»). La comparaison des deux textes analogues placés en annexe 2 rend évidents ces décalages entre le français et l'anglais. Ces textes, tirés de préfaces de dictionnaires, l'une du Petit Larousse 1998 et l'autre du Nelson Canadian Dictionary of the English Language (1997) montrent la tendance du français à choisir comme point de départ de l'énoncé un terme renvoyant à un inanimé - le dictionnaire, ses composantes ou ses caractéristiques - et à le faire suivre d'un verbe renvoyant à une activité ou à une caractéristique qui est généralement le propre d'un animé humain, ( avoir pour ambition », " répondre à vos questions », "s'adapter ", etc.), même s'il s'agit en fait souvent de métaphores puisque par "dictionnaire " on sous-entend le plus souvent "les auteurs du dictionnaire ». Par contre, dans le texte anglais, le sujet grammatical — donc, dans la plupart des cas, le terme de départ de l'énoncé - est généralement un sujet humain 
(soit le lecteur " you », soit les lexicographes «we»), et, lorsque le mot « dictionary" est choisi, il est suivi du verbe " to be ". Comme le signalent Chuquet et Paillard (1987, p.141), l'anglais a tendance à choisir comme point de départ de l'énoncé un élément animé - de préférence un animé humain - d'où la prolifération du pronom « you " —, mais non le français qui s'accommode aussi bien de non-animés ou de tournures impersonnelles. On relève également ce dernier type de décalage dans les textes de l'annexe 1 (sur les troubles de la vision) : alors que le français emploie une tournure comme "Il est tout de même bon [...] que le patient consulte l'ophtalmologiste" (deuxième paragraphe), l'anglais dit: "If you notice such problem with your eyes, see your eye doctor » (deuxième paragraphe).

\section{Modalisation de l'énoncé}

Les tendances respectives des deux langues relativement à l'homogénéité de l'énoncé expliquent que, dans l'ensemble, les textes français donnent l'impression d'être moins "statiques", plus " dynamiques " que les textes anglais. À ce décalage entre inanimé et animé, et donc entre statique et dynamique, il faut en ajouter un autre, qui tient à des différences dans la modalisation. Comme le note Maingueneau (1998, p. 87), un énoncé contient forcément des marques de modalité:

... ne serait-ce que par le mode du verbe (indicatif, subjonctif en particulier) qui indique quelle attitude l'énonciateur adopte à l'égard de ce qu'il dit ou quelle relation il établit avec le co-énonciateur à travers son acte d'énonciation.

La modalité s'exprime non seulement par les temps, mais aussi par les auxiliaires modaux (pouvoir, devoir, etc. / can, may, must, etc.), et aussi par des adverbes (probablement, peut-être / probably, maybe), ou encore par des tournures lexicales impersonnelles (il est interdit de, il est probable que / it is forbidden to, it is likely that). Au fait que l'anglais a plus souvent que le français recours aux auxiliaires pour exprimer la modalité, il faut mentionner que le français tend à être plus catégorique, moins nuancé dans le choix des supports de la modalité. Cette différence est due en partie à l'usage fréquent en anglais des auxiliaires modaux can et may, là où en français l'idée de possibilité est souvent implicite ou éliminée ${ }^{11}$ et l'idée d'interdiction beaucoup plus explicite. C'est ainsi que « Can you hear me? " se traduit normalement par « Vous m'entendez? " (Chuquet et Paillard, 1987, p. 128), l'auxiliaire can précédant un verbe de perception anglais disparaissant habituellement en français. Et il arrive souvent que l'interdiction se traduise en anglais non pas par l'auxiliaire must à la forme affirmative, mais par may à la forme négative, tournure atténuée de must qui semble bien « douce » par comparaison avec la formule courante en français « il est interdit 
de ». L'anglais donne donc l'impression d'être bien moins péremptoire que le français. C'est ce décalage qu'illustre cet extrait des directives indiquées au dos des cahiers d'examen de l'Université d'Ottawa :

Il est interdit de se présenter à la salle d'examen ou d'en sortir en possession d'un cahier d'examen / Candidates may not take any examination booklet into or away from the examination room.

L'idée d'obligation, « normalement » traduite par must en anglais et par " devoir » en français est souvent atténuée dans les textes anglais par rapport aux textes français : on relève ainsi de nombreux cas dans lesquels le français emploie l'auxiliaire " devoir » au présent, mais l'anglais non pas must, mais should. Cet auxiliaire, comme l'indiquent Leech and Svartvik (1975, p. 144), exprime une obligation à laquelle il est possible de se soustraire ( an obligation which may not be fulfilled»). Ainsi, la phrase "Articles should be under 750 words" se rend naturellement en français par "Les articles ne doivent pas dépasser 750 mots. " (Chuquet et Paillard, 1987, p.118). Cette divergence explique le ton péremptoire des textes français et le ton plus nuancé, plus circonspect des textes anglais. Un dernier exemple pour illustrer cette différence : dans une publicité, on lit en anglais "Your passenger's safety should never be an option ", et le français " La sécurité du passager, une option ? Jamais de la vie ! " (Publicité ACURA, Berline Legende 1993 ; c'est nous qui soulignons). Ici, l'obligation, traduite par should en anglais, est traduite en français par des moyens lexicaux de nature à balayer toute objection. Ainsi que le notent Chuquet et Paillard (1997, p. 118), par rapport au français, il arrive souvent que l'anglais introduise " un degré de plus dans la modalité hypothétique ".

Un autre décalage est lié à cette différence de degré d'hypothétique : alors que l'anglais présente volontiers la contingence sous l'angle hypothétique, le français tend à la présenter comme une vérité valable en tout temps. C'est ce que l'on remarque, par exemple, dans certaines annonces publicitaires rédigées en français : une possibilité/éventualité/hypothèse y est exprimée sous la forme d'une assertion, comme dans cette phrase tirée d'une « fiche » du Crédit lyonnais (banque française) : «Vous nous confiez vos opérations .... À partir du 21 septembre 1998, le Crédit Lyonnais récompense votre fidélité." Si le français transforme aisément une hypothèse en réalité, l'anglais a moins souvent recours à ce procédé. Guillemin-Flescher (1981, p. 244) commente l'exemple suivant tiré d'un texte publicitaire : "Vous aimez la musique classique, vous êtes amateur d'oeuvres d'art et vous appréciez le style rustique ou Louis XIII. Guy Degrenne a créé pour vous [...]. " Selon l'auteur, en anglais, il serait préférable de supprimer l'assertion qui pose une hypothèse comme réalité et de traduire vous par those who like : «For those who like art and classical music, [...] Guy 
Degrenne ... » Dans d'autres cas où le français comporte l'expression d'une situation éventuelle, l'anglais expliciterait la modalité hypothétique par des formules telles que Suppose/If you ... (Guillemin-Flescher, 1981, p. 245)

Enfin, il faut tenir compte d'une autre différence dans la modalité hypothétique lorsque l'on rédige dans les deux langues : alors que l'anglais exprime une prédiction/ éventualité par l'auxiliaire modal " will " ${ }^{12}$, le français, dans ce cas, emploie le temps présent qui transforme ici encore une éventualité en vérité générale. Ce décalage se remarque entre autres dans la phrase suivante qu'on lit régulièrement dans les vestiaires : "The management will not be responsible for lost articles/ La direction n'est pas responsable des objets perdus ». Citant cet exemple, Vinay et Darbelnet (1958, p. $131)$ signalent que «le français préfère l'absolu au contingent ». Le dictionnaire français-anglais Robert \& Collins Senior à l'article «will» donne, dans la section d) après la note « (indicating habit, characteristic: gen. present tense in French)» les deux exemples suivants : « this bottle will hold one litre / cette bouteille contient un litre ou fait le litre ; the car will do $150 \mathrm{~km} / \mathrm{h} /$ cette voiture fait $150 \mathrm{~km} / \mathrm{h}$ ". Comme le notent Chuquet et Paillard (1987, p. 117), le présent français correspond au modal anglais will dans un contexte générique. C'est ainsi qu'ils traduisent "All grammatical words will lose their stress when they are combined together to form an utterance" (G. Brown, Listening to Spoken English, Longman, 1977) par "Tous les mots grammaticaux perdent leur accent quand ils se combinent pour former un énoncé ". Les auteurs ajoutent que le futur est possible en français pour exprimer « le caractère prévisible, car habituel, d'un procès ", mais à certaine condition ${ }^{13}$. Et ils donnent l'opposition suivante : "All grammatical words will lose their stress when:perdent / $\boldsymbol{A}$ grammatical word will lose its stress when... : perdra. " (C'est nous qui soulignons.)

Si Demanuelli et Demanuelli (1991) affirment qu'une bonne maîtrise des problèmes concernant la modalité est essentielle en traduction, qu'il faut savoir que l'explicitation modale est plus fréquente en anglais qu'en français, que le français a recours volontiers à des supports lexicaux et l'anglais à des auxiliaires, que le français a tendance à ne pas s'embarrasser de nuances, la connaissance de ces décalages est aussi essentielle en rédaction bilingue. Les deux courts extraits de l'annexe 3 , tirés de deux ouvrages parallèles, The Canadian Style et le Guide du rédacteur, et portant sur le «Plain Language» et "La langue claire et simple " illustrent la différence de ton qui résulte de ces décalages. Le texte français semble beaucoup plus péremptoire que le texte anglais. En français, on ne s'adresse qu'indirectement au lecteur (emploi de verbes à l'infinitif), l'obligation est exprimée de façon impérative par l'auxiliaire « devoir » au présent et par une tournure lexicale catégorique ("il ne s'agit pas ... ") ; en 
anglais, on s'adresse directement au lecteur (emploie du pronom you), l'obligation est exprimée sous une forme atténuée par l'auxiliaire should, et le résultat est donné comme possible, mais non certain (emploi de l'auxiliaire can).

\section{Conclusion}

Ce survol de quelques-uns des décalages qui existent entre l'anglais et le français montre que, quel que soit le genre de textes que l'on rédige, il faut savoir adopter des stratégies stylistiques différentes lorsqu'on passe d'une langue à l'autre. Ce n'est pas toujours aisé en pays bilingue, comme le prouve l'examen de certains textes rédigés au Canada en français, et qui, bien que syntaxiquement corrects, dégagent une impression étrange. Ils sont en effet, comme certains textes traduits, imprégnés de ce que J. Delisle (1998, p.10), dans un article portant sur la traduction et intitulé «Les anglicismes insidieux ${ }^{14}$, qualifie de " mimétisme linguistique ". Pour J. Delisle, ces " anglicismes insidieux " portent atteinte au "génie de la langue ", le génie d’une langue signifiant:

... ses tendances, ses préférences pour certaines tournures ou alliances de mots, sa façon d'articuler un message, de présenter une idée, bref, tout ce qui se situe au-delà du lexique lui-même, ou plus précisément, tout ce qui conditionne les moyens d'expression et que l'usage a consacré. (Delisle, 1998, p.10)

Mais, comment savoir ce que l'usage de la langue française a consacré et empêcher qu'une langue déteigne sur l'autre ? Comme nous l'avons dit, les ouvrages de stylistique ou de grammaire unilingues ne le disent pas explicitement. Les traducteurs sont relativement gâtés puisque des ouvrages de linguistique contrastive peuvent les guider. Quant aux rédacteurs professionnels bilingues, nous pensons que la connaissance des grandes tendances propres à l'anglais et au français dégagées par les spécialistes de la traduction est de nature à les aider à opérer des choix stylistiques en fonction de la langue dans laquelle ils rédigent et à prévenir un mimétisme linguistique qui les ferait écrire en français en suivant la démarche de l'anglais et vice versa.

\section{Notes}

1 Peu d'ouvrages de style ou de grammaire de langue française indiquent ces tendances. Comme le dit Guillemin-Flescher (1986), « il y a [...] une grammaire intériorisée, un comportement langagier collectif qui apparait à travers la diversité des traductions ». 
Signalons, entre autres, La traduction : de l'anglais au français (M. Ballard, 1987), "The Word against the Word". Initiation à la version anglaise (F. Grellet, 1985) et Initiation au thème anglais. The Mirrored Image (F. Grellet, 1985), La traduction raisonnée (J. Delisle, 1993).

" Parmi les autres raisons qui donnent l'impression que le texte est «travaillé ", on peut signaler que l'anglais a recours à des phrases simples et courtes, à peu de signes de ponctuation (à part le point, on ne repère que des virgules, mais en petit nombre), alors que le français a recours plusieurs fois aux deux points, et aussi à la mise en relief à l'aide de l'introducteur " c'est ... qui ".

4 Selon Blois et Bar (1975, p.371-2), « ''organisation de la phrase est régie par trois facteurs qui combinent leurs effets $»$ : l'ordre grammatical, l'ordre logique et l'ordre affectif (mise en relief). Et les auteurs affirment à propos de l'ordre logique :

On peut justifier, par des exigences dépendant du sens, certaines habitudes qui veulent que, généralement,

a) Les phrases commencent par des compléments circonstanciels de temps et de lieu $[\ldots]$

b) L'hypothèse et l'opposition s'expriment avant le fait [...]

c) Le but, la conséquence et la comparaison sont formulés après l'expression du fait $[$...]

Il faut ajouter que des raisons esthétiques (harmonie et équilibre) jouent aussi un rôle dans l'organisation de la phrase. Rappelons à cet égard qu'est « bien caractéristique de la phrase française la tendance à sérier la phrase par masses croissantes pour une raison à la fois logique et musicale. " (Cressot, 1947, p. 210)

5 C'est pourquoi, en français, on évite de terminer une phrase qui reproduit les propos de quelqu'un ou présente des faits rapportés par l'élément incident qui donne la source de ces renseignements. Et, les phrases que l'on entend souvent au Canada et qui se terminent par " de dire X ", sentent fortement l'anglais (said so and so).

6 "Au Japon cependant l'accélération rapide de la demande interne a été induite par le vif essor des dépenses d'investissement en capital fixe, notamment dans le secteur du logement ; elle a reflété l'influence de la politique monétaire expansionniste menée par les autorités et la faiblesse des taux d'intérêt nominaux, ainsi que les allégements fiscaux et l'amélioration sensible des bénéfices des entreprises. "(Chassigneux, 1991, p. 72)

7 C'est ce qui fait dire à Claude Duneton (1978, p.142-3), dans Parler croquant, ouvrage qualifié de "manifeste souriant et plein de verve contre la langue française d'aujourd'hui » ( $4^{c}$ de couverture): "Ainsi, peu enclin à s'épancher, l'anglais annonce tout de suite l'essentiel, laissant les commentaires venir par surcroît, et parfois comme à regret dans un fléchissement de la voix. On peut imaginer que c'est là le langage des gens pressés d'exprimer ce qu'ils ont à dire, et qui préferent annoncer d'abord les choses importantes, par crainte de ne pas avoir le temps d'achever leur phrase [ ... ]. mais il est certain que la langue française demande plus de temps et de détachement pour acheminer l'auditeur vers le "but de l'énoncé". En rejetant son propos vers la fin, le Français crée chaque fois une petite attente, un léger suspense, qui confine parfois à la coquetterie, au "devinez ce que je vais dire", ce qui caractérise parfaitement le ton et les manières de salon [...] je tiens qu'un tel génie n'a pu se 
développer que chez un peuple oisif, qui a tout son temps pour classer les éléments du message dans sa tête d'abord, puis de les développer à loisir pour des auditeurs qui n'ont rien d'autre à faire qu'à l'écouter. "

8 Strunk and White, dans The Elements of Style, notent (p.26): : The proper place in the sentence for the word or group of words that the writer desires to make most prominent is usually the end. »Ils ajoutent que ce principe vaut pour les mots d'une phrase, les phrases d'un paragraphe et les paragraphes d'un texte. On trouve les mêmes règles dans Leech et Svartvik (1975, p.175) avec les deux principes de " end-focus» (« the new or most important ideas in a piece of information should be placed towards the end ") and " end-weight " ( " the more "weighty" part(s) of a sentence should be placed towards the end "), cette dernière considération dépendant de la longueur des mots ou de la complexité grammaticale. Cependant, il semble que, même si « the final position in a sentence or clause is, in neutral circumstances, the most important " (p.176), l'anglais applique souvent la seconde règle de Leech et Svartvik qui mentionnent : « But the first position is also important for communication, because it is the starting point for what the speaker wants to say, "

9 C'est pourquoi Hiromichi Uchiyama propose, dans son article intitulé « Problems caused by word order when interpreting/translating from English into Japanese : the effect of the use of inanimate subjects in English " (META, XXXVI, 2/3, 1991) des procédés de conversion. Ainsi « The avalanche destroyed several houses " devient " Because of the avalanche, several houses were destroyed ": dans l'énoncé ajusté - et traduisible littéralement en japonais -, l'objet de l'action de l'énoncé original (houses) devient le sujet d'une forme passive et le sujet devient la cause.

13 C'est ainsi que, dans la Stylistique française de Legrand (1968), les premiers exercices conseillent de substituer aux verbes être, se trouver, il y $a$ " un verbe intransitif ou pronominal de nature à faire image " et proposent, par exemple, d'écrire "Sur ses lèvres décolorées erre un sourire mélancolique " (p. 9) ou encore "À l'occident se déploie une chaîne de montagnes " (p.10), au lieu de " est ", « se trouve " ou « il y a " (un sourire, une chaîne).

" Au Canada, la fréquence du verbe « pouvoir » devant un infinitif est due, à notre avis, à l'influence de l'anglais.

12 Leech and Svartvik (1975, p. 131) notent: "Will can also be used in a habitual sense, to express the idea of 'predictability' or 'characteristic behaviour':

Accidents will happen.

A lion will only attack a human being when it is hungry."

Dans ce type de phrase, le français utiliserait le présent de l'indicatif.

13 "Le futur est ici possible à condition qu'un certain seuil d'actualisation/particularisation soit atteint, qui se manifeste au niveau de la détermination nominale. » ( p.117)

14 Dans cet article, paru en 1987 puis présenté de nouveau en 1998 dans l'Actualité terminologique, J. Delisle, après avoir mentionné entre autres qu'en français on pratique "l'économie par évidence » (le français laisse implicite ce que l'anglais explicite), on utilise « on » là où, plus familièrement, l'anglais emploie « you », signale l'une des conséquences du " mimétisme abusif ": la traduction systématique de «should " par « devoir », alors qu'un infinitif suffit en français. Il donne l'exemple suivant : 
Changes to the record should be reported in the first block.

Inscrir e les changements à apporter au dossier dans la première case. (Comparez: « les changements doivent être apportés ».)

\section{Références}

Ballard, M. (1995). " La traduction de la conjonction "and" en français ». Relations discursives et traduction. Lille, Presses universitaires de Lille, p. 221-93.

Ballard, M. (dir.) (1993). Relations discursives et traduction. Lille, Presses universitaires de Lille.

Ballard, M. (1993). La traduction à l'université. Recherches et propositions didactiques Lille, Presses universitaires de Lille.

- (1987). La traduction: de l'anglais au français. Paris, Nathan.

Ballard, M. (dir.) (1984). La traduction : de la théorie à la pratique. Lille, Presses universitaires de Lille.

Blois, J. et M. Bar (1975) (3e éd.). Notre langue française. Grammaire. Didier, Bruxelles.

Bonnard, H. (1981). Procédés annexes d'expression. Paris, Magnard.

Chassigneux, A. (1991). « Avant la charrue, les boeufs. La mise en relief du sujet et/ ou du prédicat dans la traduction de textes économiques ». Palimpsestes, $n^{\circ} 5$, vol. 1, p. $71-5$; vol. 2 , p. 5-6.

Chuquet, H. et M. Paillard (1987). Approche linguistique des problèmes de traduction anglais francais. Paris, Ophrys.

Cressot, M. (1969) (6 éd.). Le style et ses techniques. Paris. Presses universitaires de France.

Delisle, J. (1998). «Les anglicismes insidieux ». L'Actualité terminologique, vol. 31, 3, p. $10-4$.

- (1993). La traduction raisonnée. Ottawa, Presses de l'Université d'Ottawa, Collection " Pédagogie de la traduction ».

Demanuelli, C. (1995). " La virgule en question ". Relations discursives et traduction. Lille, Presses universitaires de Lille, p. 121-40.

Demanuelli, C. et J. Demanuelli. (1991). Lire et traduire. Anglais français. Paris, Masson. 
Duneton, C. (1978) (2éd.). Parler croquant. Paris. Stock + Plus.

Grellet, F. (1993). Initiation au thème anglais. The Mirrored Image. Paris, Hachette.

Guillemin-Flescher, J. (dir.) (1992). Linguistique contrastive et traduction. Paris, Ophrys.

Guillemin-Flescher, J. (1986). « Le linguiste devant la traduction ». Fabula, 7, p. 59-68.

—. (1981). Syntaxe comparée du français et de l'anglais. Problèmes de traduction. Paris, Ophrys.

Leech, G. and J. Svartvik (1975). A Communicative Grammar of English, London, Longman.

LeGrand, E. (1970). Méthode de stylistique française. Paris, Gigord.

Mainguenuau, D. (1998). Analyser les textes de communication. Paris, Dunod.

Quillard, G. (1994). "Étude de certaines différences dans l'organisation collective des textes pragmatiques anglais et français ». Babel, 43, 4, p. 313-30.

Quillard, G. et G. Akhras (1996).« And/et. Analyse distributionnelle de la conjonction copulative en français et en anglais ». META, 41,3, p. 459-70.

Strunk, W. and E.B. White (1979). The Elements of Style. New York, MacMillan.

Uchiyama, H. (1991). “Problems caused by word order when interpreting/ translating from English into Japanese: the effect of the use of inanimate subjects in English." META, 36, 2/3, p. 404-13.

Vinay, J.P. et J. Darbelnet (1995). Comparative Stylistics of French and English. Translated and edited by J.C. Sager and M.J. Hamel. Amsterdam, Philadelphia, Benjamins.

— (1958). Stylistique comparée du français et de l'anglais. Paris, Didier. 


\section{ANNEXE 1 (Les mots en gras indiquent des enchâssements ou des éléments dont la place déroge à l'ordre canonique.)}

\section{Comment commence un décollement de rétine?}

À l'intérieur de l'oeil, derrière le cristallin et l'iris, se trouve le vitré, gélatine transparente, qui donne la solidité à l'oeil et qui est contenu dans un sac appelé hyaloide. Ce sac repose sur la rétine en arrière : en principe, sans adhérer à celui-ci. Cependant, assez souvent, des adhérences anormales se produisent au cours de la vie, entre hyaloide et rétine, avec l'âge ou pour des raisons pathologiques. Comme tous les tissus humains, le vitré se déshydrate et hyaloide et rétine se séparent l'une de l'autre : c'est le décollement postérieur du vitré qui a lieu un jour ou l'autre chez $100 \%$ des individus.

Dans la majorité des cas, rétine et hyaloide n'adhérent pas l'une à l'autre, la séparation se produit sans aucune conséquence hormis quelques petites mouches volantes dites « corps flottants ». Mais il est tout de même bon, devant l'apparition brutale de mouches volantes et de filaments, que le patient consulte l'ophtalmologiste pour un examen très poussé et soigneux de la périphérie rétinienne. Lui seul pourra affirmer qu'il n'y a aucune adhérence, donc aucun traitement à faire.

Il est par contre des cas où le divorce rétine-vitré se fait bruyamment, et où les attaches contractées entre les deux tissus refusent de se rompre, chacun tire alors de son côté et, en général, c'est la rétine qui cède et se déchire au point de traction. La bride de vitré se rétracte emportant avec elle un morceau de rétine :c'est la déchirure, aussi urgente à traiter qu'un glaucome aigu. Non traitée, elle va forcément être suivie d'un décollement de rétine qui, lui, nécessitera une grosse intervention.

Aron-Rosa, D. Vos yeux : comment les protéger, comment prévenir, corriger, traiter les troubles de la vision chez l'enfant et chez l'adulte, Paris, J.-C. Lattès, 1986, p. 124-5.

\section{Retinal Detachment}

One of the most serious retinal problems occurs when something interferes with the adherence of the retina to the choroid, and the retina pulls away. The possible causes for this are numerous, some well known and others not. Torn retinal tissue can cause detachment, or detachment itself can cause the retina to tear. Other causes can be a blow to the eye, a cyst or tumor, scar tissue, a hemorrhage, infection, or some other disease of the eye. It could even simply be a general tendency toward poor adhesion. In rare cases severe nearsightedness can predispose to detachment. And as we know, retinal detachment can be a complication of cataract surgery. 
A detached retina does not hurt. Symptoms include visual loss (especially in one area of the visual field), spots before the eyes, and light flashes. But this does not mean that anyone who experiences these symptoms has a detached retina. If you notice such problems with your eyes, see your eye doctor as soon as possible to identify the cause and begin appropriate treatment, if necessary.

Retinal detachment is serious. For one thing, it will nearly always get worse if it is not treated quickly. The detached area tends to get larger, or the retina may tear away in several places. Wherever the detachment occurs, the retinal tissue no longer functions as it should and some sight is lost.

The treatment of retinal detachment is always surgical. The aim is to reattach the retina and do everything possible to make sure it does not detach again. When the underlying cause is known, it too can be treated. But often the cause is not known or, as in severe nearsightedness, it is incurable.

Eden, John, The physician guide to cataracts, glaucoma, and other eye problems, Consumer Reports Books, N. Y. Yonkers, 1992, p. 212-3.

\section{ANNEXE 2 (Les mots en caractères gras illustrent les remarques de la section 2 : homogénéité de l'énoncé)}

\section{Au lecteur}

Depuis sa naissance, en 1905, le Petit Larousse a toujours eu pour ambition de répondre à toutes vos questions, de la manière la plus simple et la plus fiable, en utilisant les vertus conjuguées de l'écrit et de l'image. S'adaptant sans cesse à l'évolution des curiosités et au progrès des connaissances, il a ainsi accompagné des millions de lecteurs - français, francophones, mais aussi étrangers - dans leur vie quotidienne ou dans leurs études. Tout au long de son histoire, il n'a cessé d'accroître le nombre de ses articles, changeant et diversifiant ses formats, renouvelant sa mise en pages, intégrant les apports des nouvelles technologies.

L'édition 1998, véritable " refonte ", témoigne une fois encore de la permanence de la vocation du Petit Larousse comme ouvrage de référence pour tous, à l'écoute des évolutions de notre langue et de notre histoire, à l'heure de la «mondalisation ". Nos équipes éditoriales et leurs correspondants dans l'ensembles des domaines de la connaissance ont donc revu, vérifié, mis à jour, complété les nomenclatures et les contenus des articles $[\ldots]$

Ainsi, fruit d'un long et passionnant travail qui a impliqué des centaines de collaborateurs, votre nouveau Petit Larousse vous aidera à voyager dans l'univers des mots et des connaissances, tout en vous invitant à de multiples et quotidiennes découvertes.

Petit Larousse, 1998 


\section{Welcome to the Dictionary}

Welcome to the Dictionary, the most comprehensive and up-to-date Canadian dictionary available. In its 1728 pages, you will find 150,000 entries covering words, people, places, and ideas presented from a distinctly Canadian point of view.

The Dictionary is a rich representation of the English language as it is spoken and written by Canadians today. You will find not only Canadianisms, but also numerous words that, singly or in combination (riding, distinct society), have a specifically Canadian application. You will be reminded, for example, why there is no point in asking a doctor to cure Klondike fever and why you would be well advised not to take your Ski-Doo out on slob ice. In addition to the definitions, we have aimed to make sense of Canadian pronunciation and spelling, often an erratic blend of U.S. and British usages. [...]

We are confident that this Dictionary, with its wide coverage, wealth of detail, and Canadian flavour, will be your indispensable microscope and telescope to the words we use and the world in which we live.

ITP Nelson Canadian Dictionary of the English Language, 1997

\section{ANNEXE 3 (Les mots en caractères gras sont commentés dan la section 3)}

\section{La langue claire et simple}

Écrire dans une langue claire et simple, cela veut dire énoncer le message de façon que les destinataires puissent le comprendre immédiatement, dès la première lecture. Il ne s'agit pas d'adopter un style puéril ou un vocabulaire pauvre, mais d'adapter le texte aux destinataires. C'est dire que le rédacteur, tout en recherchant la clarté et la simplicité, doit opter pour un style, un ton, un niveau de langue et un vocabulaire qui varieront chaque fois selon la nature du texte et selon le public-cible.

Le guide du rédacteur, Bureau de la traduction, 1996 ( $2^{\mathrm{e}}$ éd.), p. 219.

\section{Plain Language}

The purpose of a plain-language approach in written communication is to convey information easily and unambiguously. It should not be confused with an oversimplified, condescending style. Rather, by choosing straightforward vocabulary and sentence structures and by organizing and presenting your material clearly and logically, you can save the reader time and effort and ensure that your message will be clearly understood. 
The Government of Canada calls for plain language to be used in its communications with the public:

The obligation to inform the public includes the obligation to communicate effectively. Information about government policies, programs and services should be clear, objective and simple, and presented in a manner that is readily understandable. Messages should convey information relevant to public needs, use plain language and be expressed in a clear and consistent style.

[...] Use of plain language will help ensure that your message comes across clearly and that readers take appropriate action.

The Canadian Style, A Guide to Writing and Editing, Toronto, Dundurn Press, 1998, p. 245.

* Treasury Board, "Government Communications Policy," Treasury Board Publications on CD-ROM, p.17. 